\title{
De la source au delta : itinéraires de la création de Zbigniew Herbert
}

From Source to Delta: Itineraries of Zbigniew Herbert's Creation

\section{Brigitte Gautier}

\section{OpenEdition}

\section{Journals}

Édition électronique

URL : https://journals.openedition.org/recherchestravaux/849

DOI : 10.4000/recherchestravaux.849

ISSN : 1969-6434

Éditeur

UGA Éditions/Université Grenoble Alpes

Édition imprimée

Date de publication : 1 décembre 2016

Pagination : 43-52

ISBN : 978-2-84310-338-4

ISSN : 0151-1874

Référence électronique

Brigitte Gautier, «De la source au delta : itinéraires de la création de Zbigniew Herbert », Recherches \& Travaux [En ligne], 89 | 2016, mis en ligne le 12 janvier 2017, consulté le 29 octobre 2021. URL : http:// journals.openedition.org/recherchestravaux/849 ; DOI : https://doi.org/10.4000/recherchestravaux. 849 


\section{De la source au delta : itinéraires de la création de Zbigniew Herbert}

Au XIX ${ }^{\mathrm{e}}$ siècle, la ville natale de Zbigniew Herbert, Léopol ${ }^{\mathrm{I}}$, était traversée en son centre par une petite rivière, finalement recouverte par une promenade et par l'Opéra qui ferme la perspective. D'où l'énigme proposée par l'écrivain Kornel Makuszyński à propos de la ville, édifiée sur une rivière qui n’existe pas². C'est sous ces doubles auspices, de Léopol et de la rivière invisible, que nous déroulerons le fil de la métaphore introductive. Celle-ci renvoie en premier lieu à l'image habituelle qui circonscrit une vie et une ouvre, de l'origine jusqu'à la mort. Ce qu'annonce le poème "Prédiction" (Corde de lumière, I, I37) 3 :

Toutes les lignes s'enfoncent dans la vallée de la paume dans la petite cavité où coule la petite source du destin voici la ligne de vie voyez elle file comme une flèche ${ }^{4}$

Toutefois chez Herbert, la source est aussi celle de la culture occidentale, évoquée dans les essais "Lascaux» et "Chez les Doriens" de 1962, publiés la même année dans le volume Un barbare dans le jardin's. Et le delta est également celui de l'essai éponyme de I989, publié en I993, dans Nature morte avec bride

I. Aujourd'hui Lviv, en Ukraine.

2. K. Makuszyński, Uśmiech Lwowa [Le sourire de Léopol], Cracovie, Oficyna Cracovia, 1989. I ${ }^{\text {re }}$ édition : Léopol, 1938.

3. Nous donnons ici les références des volumes parus en français aux Éditions Le Bruit du Temps. Le chiffre romain renvoie au tome des Euvres poétiques complètes et le chiffre arabe, à la page.

4. "Wszystkie linie zagtębiają się $w$ dolinie dtoni / $w$ matej jamie gdzie bije źródetko losu / oto linia życia patrzcie przebiega jak strzata" (I, I36).

5. Z. Herbert, Barbarzyńca w ogrodzie, Varsovie, Czytelnik, 1962. En français : Z. Herbert, Un barbare dans le jardin, trad. J. Lajarrige révisée par L. Dyèvre, Paris, Le Bruit du Temps, 2014 . 
et mors ${ }^{6}$. Ces deux images tracent symboliquement le parcours de l'essayiste : de la culture française et italienne à la culture hollandaise. Le Labyrinthe au bord de la mer ${ }^{7}$ qui prend place en Crète, en Grèce, en Étrurie et à Rome est le chaînon intermédiaire, dont la publication aurait dû intervenir en 1975, si la censure communiste polonaise n'en avait décidé autrement. Et l'on ne saurait oublier qu'en mathématique, delta est le symbole de la variation soit de l'écart entre la valeur finale et la valeur initiale ${ }^{8}$.

Nous allons suivre ces lignes de force et leur inscription dans l'œuvre, en rendant à la littérature classique de voyage ce qui lui appartient, puis en montrant où Herbert s'en sépare, en particulier du fait de sa détermination historique et politique, et enfin comment il élabore une nouvelle qualité d'essai, grâce aussi à sa quête formelle. Sachant que la même métaphore de la source et du delta organise la ligne ainsi tracée de la littérature classique à l'essai herbertien.

La littérature de voyage, qu'elle soit reportage, essai philosophique ou récit légendaire naît en même temps que le voyage lui-même. Les témoignages les plus anciens qui nous sont parvenus, qu'il s'agisse de Pausanias ou d'Hérodote, voire d'Homère, l'attestent : c'est la première expérience que les hommes souhaitent partager avec d'autres et qui les incite à réfléchir sur l'existence. La diversité de ces écrits, plus ou moins connus en fonction de leur intérêt ou de la notoriété de leur auteur, répond du reste à celle des buts des voyages : exploration, commerce, pèlerinage... Les artistes eux, voyagent en quête de nouvelles inspirations, nées des modèles du passé (la Grèce et Rome), de nouveaux paysages (les Amériques), de nouvelles sensations (l'Orient). Au point que les itinéraires d'artistes deviennent eux-mêmes objets de romans : il n'est que de songer à Henry James ou Edward Morgan Forster. Le grand lecteur qu'est Herbert connait évidemment la littérature de voyage. Son volume de Pausanias l'accompagne jusqu'à la toute fin de sa vie; il a lu Goethe et Chateaubriand, pour ne citer que les plus emblématiques. Son premier voyage à l'étranger, en I958, lui fait découvrir la France, l'Angleterre et l'Italie et l'incite à aborder le genre, nouveau pour lui, de l'essai de voyage, alors qu'il était jusque-là poète et dramaturge. Ses voyages suivent une logique de découvertes successives. À Paris et en France, il est fasciné par les cathédrales gothiques et les peintres italiens représentés au Louvre. Puis à la National Gallery de Londres, il découvre Piero della Francesca, auquel il consacre un essai qui

6. Z. Herbert, Martwa natura z wędzidtem, Wroclaw, Wyd. Dolnośląskie, I993. En français : Z. Herbert, Nature morte avec bride et mors, trad. Th. Douchy, Paris, Le Bruit du Temps, 2012. 7. Z. Herbert, Labirynt nad morzem, Varsovie, Zeszyty Literackie, 200o. En français : Z. Herbert, Le Labyrinthe au bord de la mer, traduction et avant-propos B. Gautier, Paris, Le Bruit du Temps, 201 .

8. Je remercie l'astronome Michel Blanc pour ses précisions quant à la valeur de delta. 
porte le nom du peintre (recueilli dans Un barbare) et au British Museum, il admire les tympans du Parthénon, dont il narre l'histoire mouvementée dans «L'Acropole» (recueilli dans Le Labyrinthe). Tout ceci l'incite à voir l'Italie pour ses peintres, et Paestum, l'ancienne colonie grecque, pour son architecture, ce qui l'entraîne ensuite, inévitablement, en Grèce et en Crète, là où tout a commencé, la sculpture et les vases, dont les décors illustrent des scènes de L'Iliade. Cet itinéraire devient une remontée aux sources de la culture et témoigne d'un besoin humain de comprendre l'origine. Le narrateur met ses pas dans les traces des archéologues qui donnent un sens à ce qui est épars et sauvent ainsi de l'oubli des pans du passé.

Fidèle à ses habitudes, Herbert commence par lire ce que d'autres ont écrit, y compris les historiens de l'art. Sa synthèse personnelle, héritée en grande partie du courant romantique européen, le conduit à privilégier le thème du pèlerinage qui connote à la fois le voyage et l'admiration, voire le caractère sacré des sites visités, et l'enrichissement spirituel du pèlerin. Dans cette optique, la culture est à la fois objet de culte et source d'inspiration pour les artistes qui arpentent l'Europe, la Grèce et la dite "Terre sainte», dans le cadre de leur Grand tour. C'est le cas d'Herbert qui écrit à un ami, en juin 1959: "Je suis donc en Italie c'est-à-dire à genoux, auprès de la source'.» Et l'essayiste Herbert s'imprègne littéralement des œuvres, qu'il est capable de contempler des heures durant. Son avidité frappe les rares et patients témoins de cette contemplation :

Herbert ne passe pas d'un tableau à un autre, il s'avère tout à coup que la moitié du monde est dans ce tableau. Herbert disait que l'on devrait consacrer autant de temps à regarder un tableau que l'artiste en a mis à le créer ${ }^{\text {ro }}$.

C'est avec la même ferveur qu'il se rend en Grèce, pour la première fois en 1964, et y retourne en 1973 et 1975 :

Je bourlingue du Mont Athos jusqu’à la Cyclade la plus éloignée, Thira soit Santorin, en train, autobus, barque, bateau, à dos d'âne, la peau tannée, affamé, en manque de sommeil, pas toujours heureux et parfois trop. Cette fois, je veux découvrir la Grèce qui n'est pas celle des touristes - et c'est parfois épuisant. J'écris tout cela pour que tu ne m’envies pas, mais de toute façon tu vas m’envier, Toi! Dévoreur de Beautér ${ }^{\prime \prime}$.

La jubilation perceptible est cependant nuancée par l'ironie distanciée qui est sa marque personnelle. Dans le voyage classique, la vue est le vecteur principal de la perception : qu'il s'agisse de paysages, d'édifices, de tableaux,

9. Z. Herbert - C. Miłosz, Korespondencja [Correspondance], Varsovie, Zeszyty Literackie, 2006, p. I5. B. Gautier traduit toutes les citations.

Io. Témoignage de J. Siwiec, dans Wierność [Fidélité], Varsovie, PWN, 20I4, p. 450.

II. Z. Herbert - J. Turowicz, Korespondencja, Cracovie, a5, 2005, p. 200. 
de jeux de lumière, d'étalages de nourriture ou d'autochtones. Les notes que prend notre voyageur pèlerin sont en fait des croquis. Ce dont témoignent les 5000 dessins, aujourd'hui répertoriés dans ses archives à la Bibliothèque nationale de Varsovie ${ }^{12}$. L'essayiste est fasciné par les procédés employés par les architectes et les sculpteurs pour orienter la perception de leurs œuvres : les corrections optiques dans l'assemblage et la disposition des colonnes, les "points de vue» (p. $\left.136^{13}\right)$, ainsi des Propylées qui «semblent avoir été un rideau de pierre, destiné à arrêter le regard " (p. I35). L'idée d'un regard délibérément guidé par l'artiste conduit le narrateur à souligner son caractère subjectif. Dans le cas des Étrusques «le point d'observation y est éloigné, apparemment inhumain" (p. 192), ce qui contribue à les rendre incompréhensibles au public contemporain. À l'inverse, le portrait que Tacite fait de son beau-père, Julius Agricola, est suspecté d'être trop flatteur (p. 227).

Herbert convoque aussi les autres sens, avec un souci affiché d'authenticité qui contredit souvent l'approche esthétisante, traditionnelle : «Dans l'air flottaient des effluves d'ail, d'huile et de mouton. L'Acropole couronnée d'odeurs d'oignons.» (p. I70) De même, il insiste régulièrement sur le vacarme qui l'entoure, a priori désagréable, mais qui témoigne ainsi de la totalité de la vie. L'une des caractéristiques de sa perception est de mêler les impressions sensorielles et émotionnelles, selon la figure de «l'attelage» : «Le vestiaire se trouvait au sous-sol. C'était une sorte de cuisine des odeurs scolaires : poussière, cuir, vêtements humides et crainte» (p. 2I2). Sa passion pour les œuvres adopte même une forme érotique, rarement rencontrée dans ce genre littéraire : «ce désir de poser mes mains, de m'unir charnellement... et d'emporter - quoi? Une image? Un frisson?» (p. I68).

Retrouver l'origine de la culture occidentale en Grèce et à Rome est un thème habituel d'essais de voyage. La particularité d'Herbert consiste à remonter plus haut, vers la source, puisqu'il s'attache à la Crète et à l'Étrurie, dont il tend à démontrer que les cultures grecque et romaine sont tributaires. Dans ce type d'ouvrage, il est d'usage de citer les savants, les historiens et les écrivains qui ont forgé l'image du pays. Herbert rend discrètement hommage aux poètes polonais qui ont guidé ses pas vers des villes : Jarosław Iwaszkiewicz pour Sienne et Czesław Miłosz pour Orvieto et au peintre Joseph Czapski qui lui a fait découvrir la peinture hollandaise. Il présente des sites ou des thèmes, médiatisés (voire vampirisés) par leurs découvreurs. Cela intervient à Cnossos

I2. Ses carnets de croquis y sont répertoriés de akc. I7 953 t. I à akc. I7 953 t. 290.

13. Nous tirons la majorité de nos références du Labyrinthe (au programme de littérature comparée de l'agrégation de Lettres modernes 2016 et 2017) et trouvons plus pratique de renvoyer aux pages de l'édition citée dans le texte même. 
avec Evans qui a reconstruit le palais, démarche redoublée par Herbert sur un plan littéraire. Sur l'Acropole, Herbert, survivant des occupations soviétique et allemande, reprend à son compte le sentiment de culpabilité qu'y avait éprouvé Freud. En Hollande, Herbert s'identifie à Fromentin, peintre, écrivain et critique d'art. D'autant que notre auteur complète ses essais par des apocryphes qui augmentent le relief de la vie hollandaise au XvII ${ }^{\mathrm{e}}$ siècle. Enfin, la civilisation romaine est médiatisée par le professeur de latin qui incarne à la fois le savoir, le courage et la détermination. La mise en abyme, systématique, enracine l'idée d'héritage et de transmission mais aussi de recréation.

De même, si Herbert s'attache à la beauté des objets, il les valorise aussi pour le témoignage qu'ils portent. La colonne de Trajan à Rome est «cette gigantesque pellicule cinématographique» (p. 240); l'invocation à la source et à la mémoire qui figure sur les tablettes de Petelia (p. 33-34) est d'une humanité dérangeante, bien propre à retenir l'attention d'Herbert. La fréquentation de l'art a pour conséquence de modifier notre perception, de nous ouvrir sur l'extérieur et sur ce qui est plus grand que nous, ce que les classiques nommaient le sublime. Dans une lettre à ses parents, datée du 3 IX 59 à Paris, Herbert écrit : «Papa a raison de dire que tout me paraîtra différent à mon retour. Je n'arrête pas de réévaluer (pas seulement l'art), ce qui est fatigant mais très positif ${ }^{14}$.» Sa situation est en effet bien éloignée du loisir des voyageurs occidentaux et ses escapades sont achetées par des efforts incessants et des privations, dont la métaphore de la nage à contre-courant est une bonne illustration.

"On nage toujours vers la source, à contre-courant; les détritus nagent avec le courant.» Cette phrase d'Herbert donne son titre à l'entretien qu'il accorde à une revue clandestine, début septembre 1980 à Varsovie ${ }^{15}$, alors que les ouvriers polonais viennent de négocier avec le pouvoir communiste la création de "Solidarité/Solidarność", premier syndicat indépendant en Europe de l'Est. Les habitants des démocraties dites populaires ne détenaient pas de passeport. Ils n'en obtenaient un, pour une durée limitée, que si la police politique ne s'y opposait pas. D'où l'incertitude et l'urgence, propres aux voyageurs de l'époque :

[...] et ce sentiment encore de voir quelque chose pour la dernière fois. Il nous accompagne toujours, nous les Polonais, dans nos voyages à l'étranger. Il faut regarder à satiété pour que cela reste toute la vie ${ }^{16}$.

I4. Z. Herbert, Korespondencja rodzinna [Correspondance familiale], Lublin, Gaudium, 2008, p. I09.

I5. Krytyka, no 8, 198I, p. 48-64, repris dans Herbert nieznany [Herbert inconnu], Varsovie, Zeszyty Literackie, 2008, p. 75-97.

I6. "Światło na murze" [La lumière sur le mur], interview de M. Sołtysik du Io avril I98I (ibid. p. II6). 
Notre auteur se sent donc investi de la mission de transmettre à ses compatriotes ce qu'il réussit à voir et à lire et se choisit un patron approprié : Hermès, dieu des voyageurs mais aussi messager des dieux et guide des âmes vers l'au-delà. Lorsque, suite à ses réflexions sur l'Acropole, l'auteur écrit «j'ai été choisi », c'est à cela qu'il pense. C'est un passeur, tout comme Monsieur Cogito est «l'intermédiaire de la liberté» dans le poème "Le jeu de Monsieur Cogito" (Monsieur Cogito, II, 26I).

La dimension d'envoyé des dieux incarnée par Hermès se traduit par l'importance que son auxiliaire, le voyageur Herbert, accorde au mystère. Nager vers la source n'implique pas seulement de se heurter à des obstacles identifiables mais aussi d'en rencontrer qui ne se laissent pas totalement appréhender. De ce point de vue, l'une des images les plus saisissantes est celle du palais de Cnossos qui tombe en poussière, à mesure que l'archéologue tente de découvrir son usage, de percer son secret (p. 42). Ce que le narrateur résume plus loin : "La Crète mystérieuse aux lèvres serrées, aux yeux clos, se défend.» (p. 54) Et les objets rencontrés sont autant d'énigmes : le disque de Phaestos, l'écriture linéaire, la langue étrusque. Pour Herbert, l'une des attributions des hommes confrontés aux dieux, et l'un des sens possibles de leur existence, est de découvrir de nouveaux mondes ou de résoudre des secrets : "Le beau combat de l'intelligence et du mystère se poursuit» (p. 206), d'augmenter ainsi le territoire de l'humain. Le mystère ultime, celui de conducteur des âmes, nous amène à la mort. Herbert, le survivant, se charge de préserver la mémoire des défunts et de retrouver leur trace. Dans la carte de Cnossos qu'il adresse à son ami Zdzisław Najder, le I5 IX 64, il note : «dommage que tu ne sois pas là pour me soutenir dans ma balade d'une tombe à l'autre ${ }^{17}$ ». Najder, qui est aussi le dédicataire de «La petite âme» de 1973, a lui-même souligné le sens de cette mission dans l'œuvre ${ }^{18}$.

La deuxième leçon de la nage à contre-courant et de la poursuite acharnée de la culture se trouve dans le projet antitotalitaire, clairement énoncé : «Nous, héritiers des crimes et des silences, tentons de rendre justice au passé, de rendre leur voix aux grands muets de l'histoire, aux peuples qui n'ont pas réussi.» (p. 187) C'est ainsi que sont convoqués les Étrusques, les Samiens, les Bretons, les Albigeois, les Templiers, tous ceux qui ont été humiliés, injustement condamnés, décimés, et de surcroît, volontairement oubliés par l'historiographie officielle. Pour cette même raison mais à l'inverse, la description que

I7. $M s$, archives privées de Z. Najder.

I8. Z. Najder, «La poésie comme devoir», dans En hommage à Z. Herbert, rééd. D. KnyszTomaszewska, B. Gautier, A. Ciesielska, Varsovie-Paris, Les Nouveaux Cahiers franco-polonais, $\mathrm{n}^{\circ} 5$, 2005, p. II3-I23. 
fait le narrateur du paysage de Sparte est étonnamment sèche et laconique, par rejet viscéral d'une cité uniquement guerrière. L'image prégnante du siège, de Montségur à Leyde, en passant par l'Acropole ou Samos, trouve aussi une parfaite illustration dans le poème "Rapport de la Ville assiégée» (Rapport de la Ville assiégée, II, 45I). Il représente la victoire de l'acharnement, de la résistance et des valeurs, contre les assauts de la tyrannie, destinée à s'effondrer, comme le prophétise la fin de «Samos» (Le Labyrinthe).

Plus généralement, en habitué de la propagande, l'auteur relit lucidement les témoignages historiques et les légendes associées. Il identifie l'intérêt politique de certains protagonistes à faire accréditer leur version, fallacieuse, des événements. Sa réappropriation de l'histoire débouche sur la question épistémologique induite, du mode de transmission. La question surgit au début très simplement avec les couleurs, en particulier les demi-teintes, qu'il conviendrait de nommer précisément quand on évoque des tableaux. Mais il est d'autres difficultés : «une description littéraire, c'est comme si on déplaçait des meubles pesants, elle se déroule dans le temps, lentement ${ }^{19}$ ». Ces interrogations, ces hésitations trouvent aboutissement, sinon solution, dans la figure du delta.

La lettre grecque reprend la forme du delta du Nil et c'est d'Égypte qu'est issue la figure de l'Hermès Trismégiste ou "trois fois grand", symbole de l'ésotérisme qui redouble ou triple en quelque sorte l'Hermès traditionnel, déjà associé à l'hermétisme et à l'alchimie, laquelle vise la transmutation de la matière. Entre autres sens, le poème "Le Voyage» (Élégie au départ, III, 69) comporte des allusions à ces tentatives et à la transformation de l'individu. Celle-ci s'applique en premier lieu à l'écriture d'Herbert ou, plus précisément, à sa création. Il s'agit de mettre en scène et de mettre en mots l'expérience du voyage. L'une des modifications de détail concerne, par exemple, le nom du bateau qui l'emmène du port du Pirée en Crète : «Égée», qu'il rebaptise "Thésée» dans «Le labyrinthe au bord de la mer». Ce qui place son itinéraire sous le signe de la conquête des Argonautes et de la légende du Minotaure, présente implicitement dans le titre de l'essai et, explicitement, dans un poème célèbre ("L'histoire du Minotaure», Monsieur Cogito, II, 219).

Chacun des essais fait l'objet d'une organisation rhétorique et dramatique précise et comporte régulièrement une scène d'exposition où le narrateur, à peine arrivé dans un lieu donné, se précipite pour admirer l'un de ses monuments, de préférence de nuit. Cela intervient à Paris, où il débarque gare de l'Est et se rend à pied à Notre-Dame, illuminée. La scène se répète à Rome, où il va au Forum (qu’il a dessiné en classe de latin) également illuminé. De même à Athènes, où il finit par apercevoir l'Acropole :

19. Z. Herbert, Nature morte avec bride et mors, ouvr. cité, p. I37. 
Une rangée de maisons, d'entrepôts, de dépôts sordides et de boutiques, recouverts d'une poussière blanche. Et soudain au-dessus d'une rue étroite, très haut, tout à fait inattendue : la voilà ${ }^{20}$

Après l'admiration, propre à chaque touriste, vient le moment où le narrateur tente de comprendre comment le monument a surgi de terre. Sa patiente reconstitution s'appuie sur différentes pièces à conviction, elles-mêmes produits de recherches approfondies. Il retrouve ainsi des cartons de dessins de l'Acropole, datant du XVII ${ }^{\mathrm{e}}$ siècle, et donc d'avant sa destruction, à la Bibliothèque nationale à Paris (p. 156). De même, il met au jour les moyens matériels de la construction des cathédrales, les mécanismes de la folie spéculatrice des tulipes ou l'organisation de l'armée, instrument de la puissance de Rome. Son investigation vise à identifier les mécanismes de la formation de l'art et plus généralement, ceux de la civilisation. Selon cette démarche, ses essais apparaissent comme miroir de la création, ce dont il s'explique indirectement dans le poème "Le miroir déambule le long du chemin " (Rovigo, III, I77), où :

il [l'art] se mesure aussi à l'histoire

avec des bonheurs divers

il tente de l'apprivoiser

de lui donner un sens humain ${ }^{21}$

Ce qui n'exclut pas le jeu avec le lecteur, ironiquement révélé par la phrase suivante :

Du plus haut sommet du mont Cynthe, on voit la couronne des Cyclades : Tinos, Andros, Syros, Kythnos, Sérifos, Antiparos, Paros, Ios, Naxos, Amorgos, Mykonos, Ikaros. Cela est un peu exagéré car l'on ne voit pas toutes ces îles, mais leurs noms sont si beaux qu'il est difficile de ne pas céder à la tentation de les énumérer ${ }^{22}$.

L'enjeu des essais est de créer une harmonie esthétique, née de l'assemblage idoine de pierres, de monuments, de phrases ou de sons qui peut être réactivée à chaque rencontre avec le spectateur ou le lecteur. D'autant que bon nombre de lecteurs partent sur les traces d'Herbert le voyageur lorsqu'ils le peuvent, souvent bien des années plus tard, après 1989 et le renversement du système communiste et l'introduction de la démocratie en Pologne. Car c'est l'un des attributs de l'écriture herbertienne que de transformer son lecteur, en l'incitant à adopter une vue critique sur l'histoire et panoramique sur le monde, puis à

20. Respectivement : Un barbare dans le jardin, ouvr. cité, p. I44 et Le Labyrinthe au bord de la mer, ouvr. cité, p. I26 et 2I4.

2I. "zmaga się także z historią/ze zmiennym powodzeniem//stara się ją oswoić/nadać ludzki sens» (III, I76).

22. Ibid., p. Io9. 
retrouver le dessein initial de l'artiste, altéré par les vicissitudes de l'histoire. Vingt ans après avoir écrit "Arles» (Un barbare), Herbert reçoit une lettre d'une archéologue polonaise qui, commentant sa métaphore de la lueur d'un phare guidant les défunts, à l'église Saint-Honorat, ressent le besoin de l'étayer scientifiquement :

quelques gravures des catacombes romaines peuvent être interprétées comme la représentation du navire-âme qui tend vers le phare - havre éternel ${ }^{23}$.

Sans surprise, dans l'œuvre et la vie d'Herbert, le delta est à la fois terme de la vie, révélation et aboutissement d'un parcours initiatique. S'il faut en croire le poème "À la rivière" (Rapport de la Ville assiégée, II, 293) :

apprends-moi à m'obstiner et à durer pour qu'à mon heure dernière je mérite de reposer à l'ombre du grand delta au triangle sacré du début et de la fin ${ }^{24}$

Il serait pourtant hasardeux d'oublier le don de mise en scène de l'auteur et la tonalité élégiaque obligée, requise dans ce genre d'œuvre. Indépendamment d'une lecture métaphysique ou hermétique, il peut simplement s'agir de l'apaisement escompté au terme d'une vie trépidante. Ce thème est également le fil conducteur de son dernier volume : l'Épilogue de la tempête $e^{25}$ qui doit beaucoup à Prospero, le magicien shakespearien. L'image du delta, où viennent se perdre un fleuve et ses différents cours d'eau, sied au dessinateur qu'était Herbert, d'autant que sa vie connut tout à la fois les aléas du hasard, de l'arbitraire et de son libre arbitre qui servirent tous à irriguer sa création. Lorsqu'il reçoit le prix T. S. Eliot en novembre 1995, alors qu'il est très malade, il écrit : "Chaque année, je fais un voyage en imagination en Grèce, pour éprouver une joie pure et atteindre la source ${ }^{26}$.» Preuve qu'à tout moment, il fut capable de s'abstraire de la souffrance, sans pour autant renoncer à la plénitude de la vie. La source et le delta ne sont donc pas des points fixes dans une biographie ou une œuvre. Et si l'on en croit Józefa Hennel : «il [Herbert] revenait toujours à ses propres mondes, nés de la source qui jusqu'au bout jaillit en lui-même ${ }^{27}$ ».

23. E. Makowiecka, 198I, ms, in akc.I8.005 t. 44, fonds Herbert, Biblioteka Narodowa, Zakład Rękopisów, Varsovie.

24. "naucz mnie rzeko uporu i trwanialabym zastużyt w ostatniej godzinie/ na odpoczynek w cieniu wielkiej delty/w świętym trójkącie początku i końca» (II, 292).

25. Z. Herbert, Épilogue de la tempête, trad. B. Gautier, Paris, Le Bruit du Temps, 2014.

26. Discours de réception du prix T. S. Eliot, novembre $1995, m s$, in akc.18 005 t. 72 , ibid.

27. J. Hennel, «Zbyszek», Tygodnik Powszechny, 32, 1998, repris dans Wierność, ouvr. cité, p. 132 . 
En I963, à la bibliothèque du British Museum, Herbert rassemble des notes de lecture avec l'idée de rédiger une sorte d'"Introduction à la théorie du voyage ", une fois qu'il sera à la retraite ou trop fatigué pour voyager. Il abandonnera cependant ce projet qui ne lui correspond pas, car il n'est ni compilateur ni théoricien et sort des sentiers battus. Il ne décrit en effet ni Athènes, ni Rome, ni Paris, ni Londres... mais s'attache à des villes mineures qui ont contribué à civiliser et faire rayonner une région. Et lorsqu'il aborde un monument ou un phénomène culturel, c'est aussi parce que l'art lui permet de comprendre les hommes de cette période, de souligner leur parenté avec les contemporains et d'introduire ainsi une forme d'éternité. Indépendamment de la puissance de sensation et d'émerveillement qui émane de ces pages, la conviction de la capacité humaine de résilience et d'accomplissement explique aussi sans doute leur écho persistant. Il n'est que de citer le poème "Le Voyage», initialement paru en octobre $1975^{28}$ mais finalement inclus dans le tome de 1990, Élégie au départ (III, 69) :

Si tu pars en voyage que ce soit un long voyage un vagabondage sans but apparent une errance à tâtons pour explorer au toucher non des seuls yeux les aspérités de la terre et te mesurer au monde de toute ta peau $[\ldots]$

Si tu sais déjà fais silence sur ton savoir apprends le monde à neuf comme un philosophe ionien jouis de l'eau et du feu de l'air et de la terre car ils subsisteront quand tout disparaîtra le voyage subsistera même si ce n'est plus le tien $[\ldots]^{29}$

28. Tygodnik Powszechny, 4I, I975, p. I.

29. "Jeżeli wybierasz się w podróż niech będzie to podróż dtuga/wędrowanie pozornie bez celu btądzenie po omacku/żebyś nie tylko oczami ale także dotykiem poznat szorstkoś́ ziemi / i abyśs cata skóra zmierzyt się ze światem [...] Jeżeli już będziesz wiedziat zamilcz swoją wiedzę/ na nowo ucz się świata jak joński filozof/smakuj wodę i ogień powietrze i ziemię/bo one pozostana gdy wszystko przeminie/i pozostanie podróż chociaż już nie twoja [...]" (III, 64). 